\title{
BMJ Open Evidence for the effectiveness of minimum pricing of alcohol: a systematic review and assessment using the Bradford Hill criteria for causality
}

\author{
Sadie Boniface, ${ }^{1}$ Jack W Scannell, ${ }^{2}$ Sally Marlow ${ }^{1}$
}

To cite: Boniface $\mathrm{S}$, Scannell JW, Marlow S. Evidence for the effectiveness of minimum pricing of alcohol: a systematic review and assessment using the Bradford Hill criteria for causality. BMJ Open 2017;7:e013497. doi:10.1136/bmjopen-2016013497

- Prepublication history and additional material is available. To view please visit the journal (http://dx.doi.org/ 10.1136/bmjopen-2016013497).

Received 15 July 2016 Revised 28 February 2017 Accepted 6 March 2017

CrossMark

\begin{abstract}
${ }^{1}$ Department of Psychology and Neuroscience, National Addiction Centre, Institute of Psychiatry, King's College London, London, UK ${ }^{2}$ School of Social and Political Science, University of Edinburgh, Edinburgh, UK
\end{abstract}

Correspondence to Dr Sadie Boniface; sadie.boniface@kcl.ac.uk

\section{ABSTRACT}

Objectives: To assess the evidence for price-based alcohol policy interventions to determine whether minimum unit pricing (MUP) is likely to be effective.

Design: Systematic review and assessment of studies according to Preferred Reporting Items for Systematic Reviews and Meta-Analyses (PRISMA) guidelines, against the Bradford Hill criteria for causality. Three electronic databases were searched from inception to February 2017. Additional articles were found through hand searching and grey literature searches.

Criteria for selecting studies: We included any study design that reported on the effect of pricebased interventions on alcohol consumption or alcohol-related morbidity, mortality and wider harms. Studies reporting on the effects of taxation or affordability and studies that only investigated price elasticity of demand were beyond the scope of this review. Studies with any conflict of interest were excluded. All studies were appraised for methodological quality.

Results: Of 517 studies assessed, 33 studies were included: 26 peer-reviewed research studies and seven from the grey literature. All nine of the Bradford Hill criteria were met, although different types of study satisfied different criteria. For example, modelling studies complied with the consistency and specificity criteria, time series analyses demonstrated the temporality and experiment criteria, and the analogy criterion was fulfilled by comparing the findings with the wider literature on taxation and affordability.

Conclusions: Overall, the Bradford Hill criteria for causality were satisfied. There was very little evidence that minimum alcohol prices are not associated with consumption or subsequent harms. However the overall quality of the evidence was variable, a large proportion of the evidence base has been produced by a small number of research teams, and the quantitative uncertainty in many estimates or forecasts is often poorly communicated outside the academic literature. Nonetheless, price-based alcohol policy interventions such as MUP are likely to reduce alcohol consumption, alcohol-related morbidity and mortality.

\section{Strengths and limitations of this study}

- This review adds to an emerging literature of systematic reviews synthesising findings using the Bradford Hill criteria for causality in research areas where traditional meta-analyses of randomised controlled trials are not possible or appropriate.

- A range of study designs were included, allowing for a comprehensive review of a disparate evidence base to investigate whether minimum unit pricing of alcohol is likely to reduce alcohol consumption and alcohol-related harm.

- Studies examining the effects of alcohol taxation or changes in alcohol affordability, or studies solely reporting on price elasticity of demand, were not included.

Methodological quality of studies was variable.

\section{INTRODUCTION}

There are many policies and programmes that aim to reduce harms from alcohol. ${ }^{1}$ One of these is minimum alcohol pricing, which exists in a number of countries around the world. The most notable example of this is Canada, where there are government monopolies on alcohol sales and a variety of types of minimum pricing in operation. For example, there is a minimum price per litre of a particular drink in British Columbia ${ }^{2}$ and a (higher) minimum price linked to drink type and strength in Saskatchewan. ${ }^{3}$ Other countries with minimum alcohol pricing include Belarus, Kyrgyzstan, the Republic of Moldova, the Russian Federation and Ukraine. ${ }^{4}$ Minimum alcohol pricing is being considered by governments in Ireland ${ }^{5}$ and has also been reviewed in Australia ${ }^{6}$ and New Zealand. $^{7}$

The situation with regards to minimum alcohol pricing in the UK is complex. 
In England and Wales, there has been a ban on alcohol being sold at below cost (the total amount of 'duty plus value added tax (VAT)') since May $2014 ;^{8}$ and the first conviction for selling alcohol below this level took place in 2016. ${ }^{9}$ Duty plus VAT is equivalent to a $70 \mathrm{cl}$ bottle of vodka $(37.5 \%$ alcohol by volume (ABV)) costing a minimum of $£ 8.72,{ }^{10}$ whereas under a minimum price of 50 pence per unit (one UK unit $=10 \mathrm{~mL}$ or $8 \mathrm{~g}$ ethanol), this would cost £13.13. In 2012, the UK coalition government cited support for minimum unit pricing (MUP) in its alcohol strategy, ${ }^{11}$ and legislation to have a minimum price of $£ 0.50$ per unit was passed in Scotland the same year. ${ }^{4}$ Following the change to a Conservative majority government in 2015, it is unclear whether there is still central government support for MUP. In Scotland, the Scotch Whisky Association challenged the 2012 legislation in the Scottish Court of Session, which referred the case to the Court of Justice of the European Union (CJEU) in 2014. ${ }^{12}$ In late 2015, the CJEU referred the case back to the Scottish courts to investigate proportionality (that the same objective cannot be met through increased taxation), ${ }^{13}$ which could have implications for other EU countries considering MUP. In late 2016, the Scottish Court of Session ruled that MUP does not contravene EU law; ${ }^{14}$ however, the Scotch Whisky Association then appealed to the UK Supreme Court. ${ }^{15}$
In light of this ongoing consideration of MUP in the UK, in this paper we assess the effectiveness of minimum alcohol price interventions to reduce alcoholrelated harm. Alcohol-related harm costs the National Health Service in England £3.5 billion each year and the estimated cost to society is $£ 21$ billion per year. ${ }^{16}$ The latest annual figures for England (population of 54 million) show over 1 million alcohol-related hospital admissions (2013/2014) and 6500 alcohol-related deaths (2013); and these figures represent increases compared with a decade previously of $115 \%$ and $10 \%$, respectively. ${ }^{16}$

We systematically review the literature on the effect of price interventions or policies such as MUP on alcohol consumption, alcohol-related morbidity and mortality, and wider harms. We use the nine Bradford Hill criteria for causality as a framework with the aim of assessing the likely effectiveness of MUP as a policy to reduce alcohol consumption and alcohol-related harm.

\section{METHODS}

A systematic literature search was performed according to Preferred Reporting Items for Systematic Reviews and Meta-Analyses (PRISMA) guidance (see figure 1 for PRISMA flow diagram and online supplementary file for excluded studies).
Figure 1 PRISMA 2009 flow diagram of studies in this systematic review. PRISMA, Preferred Reporting Items for Systematic Reviews and Meta-Analyses.
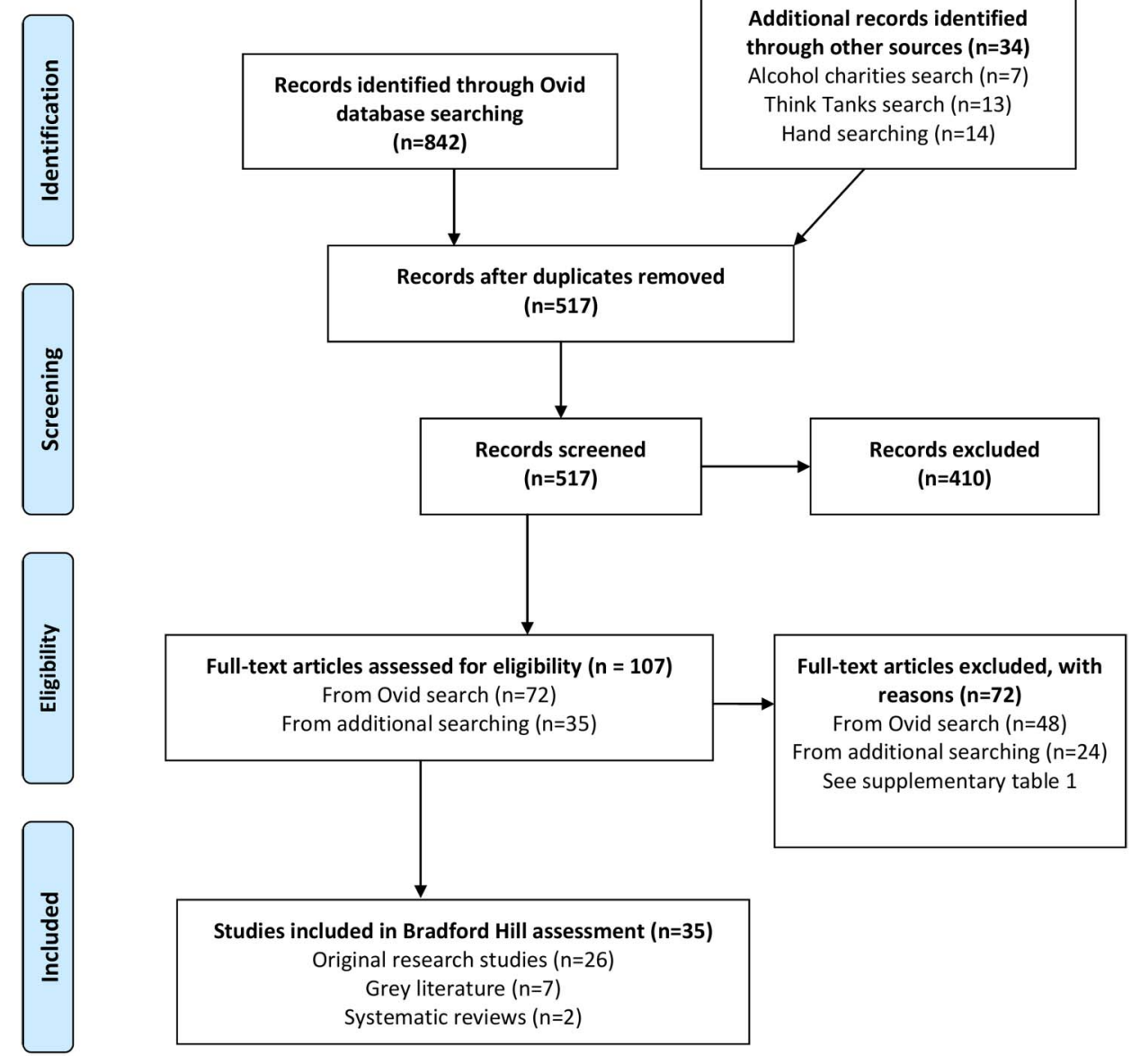
Identification of studies

Three electronic databases were searched for titles or abstracts containing 'minimum unit pric\$' OR 'minimum pric\$' OR 'floor pric\$' OR ‘pric\$ AND policy' AND alcohol. The databases were PsycINFO (1806 to February Week 2 2017), Embase (1974 to 2017 Week 07) and Ovid Medline (1946 to February Week 2 2017). We also searched the websites of five alcohol charities for publications or reports related to 'price', and also searched 20 leading UK think tanks for 'alcohol' or 'addiction'.

Inclusion criteria were: any study design; populationlevel studies exploring at least one aspect of the effect of interventions or policies leading to changes in the minimum price of alcohol, including but not limited to changes in alcohol sales, consumption, morbidity and mortality; and individual-level studies exploring minimum alcohol prices and alcohol purchasing, consumption, morbidity or mortality; written in English.

Exclusion criteria were: studies about taxation, affordability, price elasticity of demand for alcohol and general changes in alcohol price not the result of an intervention or policy (there is a large literature on each of these already and reviewing all of these studies was beyond the scope of this review); studies about public perceptions of MUP; and studies where a conflict of interest was reported in the paper, whether this was in favour of or against MUP.

All 33 studies that met the inclusion criteria were assessed against the Bradford Hill criteria for causality and the methodological quality appraised. These included 26 original research studies and seven studies from the grey literature, and in addition two systematic reviews pertinent to the analogy criterion were included. Of the 26 research studies, there were 9 cross-sectional surveys, 8 time series analyses or similar, 7 modelling studies, 1 qualitative study and 1 trial.

\section{Analysis of included studies}

Quality of included studies was assessed independently by two reviewers and using validated tools. Owing to the wide variation in study designs among the included studies, the Effective Public Health Practice Project's (EPHPP) tool was used for assessing all quantitative studies, as recommended by the Cochrane Handbook for assessing studies in public health. ${ }^{17}$ Qualitative studies $(n=1)$ and systematic reviews $(n=2)$ included in this review were not covered by the EPHPP tool and so were assessed using the Critical Appraisal Skills Programme (CASP) tools specific to these study designs.

Nine criteria to determine causality were suggested by Bradford Hill in an influential 1965 paper. ${ }^{18}$ Increasingly, the Bradford Hill criteria are a standard framework to assess the impact of interventions where it is not ethical or practical to conduct randomised controlled trials (RCTs). Our interpretation of the Bradford Hill criteria for the purpose of this review is listed in table 1. Two reviewers assessed each study against each of the nine criteria and agreed which studies provided relevant evidence for or against each criterion.

\section{RESULTS}

The included studies that are published in peer-reviewed journals (26 research studies and two systematic reviews) are listed by study type in table 2 with information on study characteristics and methodological quality. Of the research studies, the methodological quality was rated as 'strong' in 15 studies, 'moderate' in 8 studies and 'weak' in 3 studies. Both of the systematic reviews were rated 'strong'. The seven reports from the grey literature are listed in table 3. Five of the seven were rated as of 'strong' methodological quality, with the remaining two not appropriate to rate using our critical appraisal tools.

\section{Consideration against the Bradford Hill criteria for determining causality \\ Strength of the association}

In 16/26 studies published in peer-reviewed journals, strength of the association between pricing and alcohol consumption or alcohol-related harm was evidenced by a summary statistic such as an OR, and by a test of the statistical significance of the association. As well as the statistical significance of the summary statistic, the magnitude of the effect was also considered, as a larger effect size corresponds to a greater population health impact. Studies in Canada found that $10 \%$ increases in minimum prices were associated with reductions in alcohol consumption of $3.4-8.4 \%,{ }^{2}{ }^{3}$ reductions in alcohol-attributable hospital admissions of $9 \%^{21}$ and reductions in alcohol-related mortality of $32 \%$, each of which was statistically significant. ${ }^{24}$ Cross-sectional studies in the UK, Ireland, Australia ${ }^{32-36}{ }^{38-40}$ and one trial from the USA ${ }^{41}$ found statistically significant associations between cheaper alcohol and heavier drinking. The magnitude of the association varied across these studies, but due to different study measures and outcomes, the results are not all comparable. As an indication, the OR for buying alcohol below a proposed minimum price among heavier drinkers was 1.34 in Crawford's study, ${ }^{35} 1.50$ in Cousins's study ${ }^{34}$ and 1.70 in Callinan's study. ${ }^{33}$ There was not any evidence to support this criterion from the grey literature. Overall, there is reasonably good support for the strength of the association criterion.

\section{Consistency}

This criterion requires looking across all the studies included in the review to see whether similar conclusions have been drawn. Inverse associations between alcohol pricing and alcohol consumption or harm have been documented in countries in Europe, North America and Australia, and although most studies are from the last 10 years, there are studies from the 1970s and 1980s as well. There is evidence from different research teams and different types of study including 
Table 1 Bradford Hill criteria for assessing causation and the definitions used in this review

\begin{tabular}{|c|c|c|}
\hline Criterion & Bradford Hill criteria (1965) & Application in this review \\
\hline $\begin{array}{l}\text { 1. Strength of the } \\
\text { association }\end{array}$ & $\begin{array}{l}\text { The strength of a supposed association between an } \\
\text { intervention and an outcome is determined by the } \\
\text { appropriate statistic used to measure the protective } \\
\text { effect of an intervention (eg, relative risk or OR). } \\
\text { This is the most important factor determining } \\
\text { causation }\end{array}$ & $\begin{array}{l}\text { A statistically significant change }(p<0.05) \text { in alcohol } \\
\text { consumption or alcohol-related harms, in the } \\
\text { expected direction. The exact magnitude of the } \\
\text { association was assessed on a study by study } \\
\text { basis }\end{array}$ \\
\hline 2. Consistency & $\begin{array}{l}\text { Has it been repeatedly observed by different } \\
\text { persons, in different places, circumstances and } \\
\text { times? }\end{array}$ & $\begin{array}{l}\text { Whether different studies conducted in different } \\
\text { locations, in different populations, by different } \\
\text { investigators and at different times have reported } \\
\text { similar findings }\end{array}$ \\
\hline 3. Specificity & $\begin{array}{l}\text { Specificity is present when the intervention is } \\
\text { exclusive to the outcome and when the outcome } \\
\text { has no other known cause or associated risk } \\
\text { factors; cautions that this criterion should not be } \\
\text { overemphasised and that if specificity is not } \\
\text { apparent, this does not preclude causation }\end{array}$ & $\begin{array}{l}\text { If pricing was the only reason that alcohol } \\
\text { consumption or alcohol-related harm could have } \\
\text { fallen, this adds to the argument for causality. } \\
\text { However, if a price intervention was one of a } \\
\text { number of alcohol policy interventions, then this } \\
\text { criterion is not satisfied }\end{array}$ \\
\hline 4. Temporality & $\begin{array}{l}\text { Refers to temporal relationship of association } \\
\text { between exposure and disease outcome; to infer } \\
\text { causality, exposure must precede outcome }\end{array}$ & $\begin{array}{l}\text { e pricing intervention studied must have taken } \\
\text { ace before a change in alcohol consumption or } \\
\text { irm was observed }\end{array}$ \\
\hline $\begin{array}{l}\text { 5. Dose- } \\
\text { response }\end{array}$ & $\begin{array}{l}\text { If the association is one in which a dose-response } \\
\text { curve or biological gradient can be observed, this } \\
\text { adds to the case for causality }\end{array}$ & $\begin{array}{l}\text { If interventions leading to a larger increase in prices } \\
\text { had a greater effect on alcohol consumption and } \\
\text { alcohol-related harm than interventions where the } \\
\text { price change was small, or if studies demonstrate } \\
\text { that different minimum prices have differing effects, } \\
\text { in the expected direction }\end{array}$ \\
\hline 6. Plausibility & $\begin{array}{l}\text { A likely biological mechanism linking the } \\
\text { intervention to the observed findings helps to } \\
\text { explain causality; plausibility depends on biological } \\
\text { knowledge of the day }\end{array}$ & $\begin{array}{l}\text { Studies that found an association between price } \\
\text { and population-level alcohol consumption and that } \\
\text { heavier drinkers tend to purchase the cheapest } \\
\text { alcohol could demonstrate plausibility }\end{array}$ \\
\hline 7. Coherence & $\begin{array}{l}\text { When the evidence from different disciplines } \\
\text { sources 'hangs well together' and does not conflict } \\
\text { with other generally known facts, this criterion is } \\
\text { met }\end{array}$ & $\begin{array}{l}\text { Describes whether studies conducted in different } \\
\text { settings or disciplines had complementary findings. } \\
\text { Will not be demonstrated by a single study in } \\
\text { isolation but rather the evidence base as a whole }\end{array}$ \\
\hline 8. Experiment & $\begin{array}{l}\text { Experimental evidence from laboratory studies or } \\
\text { RCTs could potentially provide strongest support for } \\
\text { causation } \\
\text { This criterion often provides the strongest support }\end{array}$ & $\begin{array}{l}\text { In addition to laboratory studies and RCTs, natural } \\
\text { experiments with before-and-after measures could } \\
\text { also show the effectiveness of minimum unit pricing } \\
\text { in a 'real-world' setting }\end{array}$ \\
\hline
\end{tabular}

cross-sectional studies, time series analyses and econometric modelling studies. Support for the consistency criterion is very strong.

\section{Specificity}

The specificity criterion relates to whether changes in alcohol consumption or harm could be attributed to anything other than the price intervention. Many studies included have statistically adjusted for confounding factors; however, the best support for the specificity criterion comes from the econometric modelling studies because there is no risk of residual confounding. The
Sheffield Alcohol Policy Model is one such model and has been applied in England, ${ }^{25-29}{ }^{47}$ Scotland $^{45} 51$ and Canada $^{48}$ and provides very strong support for the specificity criterion. Further support is provided by other different modelling studies in the $\mathrm{UK}^{49}{ }^{50}$ and Australia ${ }^{30} 31$ and a (non-randomised) trial in the USA. ${ }^{41}$ Thus, support for the specificity criteria is very strong.

\section{Temporality}

It is important that pricing interventions take place before changes to alcohol consumption and harm to attribute causality. Strong support for this criterion 


\begin{tabular}{|c|c|c|c|c|c|c|c|c|c|c|}
\hline \multirow[b]{2}{*}{ Study type } & \multirow[b]{2}{*}{$\begin{array}{l}\text { First author } \\
\text { and year } \\
\text { published }\end{array}$} & \multicolumn{5}{|c|}{ Study characteristics } & \multicolumn{3}{|c|}{ Study assessment } & \multirow[b]{2}{*}{$\begin{array}{l}\text { Bradford Hill } \\
\text { criteria met }\end{array}$} \\
\hline & & Country & Study design & $\begin{array}{l}\text { Population or } \\
\text { participants }\end{array}$ & $\begin{array}{l}\text { Pricing intervention } \\
\text { studied }\end{array}$ & Outcomes studied & $\begin{array}{l}\text { Peer } \\
\text { reviewed }\end{array}$ & $\begin{array}{l}\text { Conflict } \\
\text { of } \\
\text { interest }\end{array}$ & $\begin{array}{l}\text { Quality } \\
\text { rating }\end{array}$ & \\
\hline \multirow[t]{6}{*}{$\begin{array}{l}\text { Natural } \\
\text { experiments } \\
\text { and time } \\
\text { series } \\
\text { analyses }\end{array}$} & $\begin{array}{l}\text { Bhattacharya } \\
2013^{19}\end{array}$ & Russia & $\begin{array}{l}\text { Time series } \\
\text { analysis of } \\
\text { panel data set }\end{array}$ & $\begin{array}{l}\text { Populations of } 77 \\
\text { Russian oblasts } \\
\text { (provinces), 1970- } \\
2000\end{array}$ & $\begin{array}{l}\text { Substantial increases in } \\
\text { administratively set alcohol } \\
\text { prices } 1985-1988, \text { along } \\
\text { with six other antialcohol } \\
\text { measures }\end{array}$ & Mortality & Yes & $\begin{array}{l}\text { Not } \\
\text { stated }\end{array}$ & Strong & $\begin{array}{l}\text { SA, CON, TE, } \\
\text { PL, CO, EX, }\end{array}$ \\
\hline & $\begin{array}{l}\text { Herttua } \\
2015^{20}\end{array}$ & Finland & $\begin{array}{l}\text { Time series } \\
\text { analysis }\end{array}$ & $\begin{array}{l}\text { General population } \\
\text { using population } \\
\text { registry }\end{array}$ & $\begin{array}{l}\text { Modelled } 1 \% \text { increase in the } \\
\text { average minimum price of all } \\
\text { alcoholic beverages based } \\
\text { on actual price increases } \\
\text { adjusted for inflation using } \\
\text { Consumer Price Index }\end{array}$ & $\begin{array}{l}\text { Alcohol-related } \\
\text { mortality }\end{array}$ & Yes & None & Strong & $\begin{array}{l}\text { SA (not } \\
\text { universal } \\
\text { findings- } \\
\text { subgroup only), } \\
\text { CON (counter } \\
\text { findings) TE, } \\
\text { PL, CO, EX }\end{array}$ \\
\hline & $\begin{array}{l}\text { Stockwell } \\
2012^{2}\end{array}$ & Canada & $\begin{array}{l}\text { Cross-sectional } \\
\text { versus time } \\
\text { series analysis } \\
\text { of ecological } \\
\text { data }\end{array}$ & $\begin{array}{l}\text { Population of British } \\
\text { Columbia }\end{array}$ & $\begin{array}{l}\text { Actual minimum price } \\
\text { increased over a } 20 \text {-year } \\
\text { period. Study modelled a } \\
10 \% \text { increase in the average } \\
\text { minimum price of all } \\
\text { alcoholic beverages adjusted } \\
\text { by monthly Consumer Price } \\
\text { Index }\end{array}$ & $\begin{array}{l}\text { Alcohol } \\
\text { consumption } \\
\text { (measured by sales) }\end{array}$ & Yes & None & Strong & $\begin{array}{l}\text { SA, CON, TE, } \\
\text { DR, CO, EX }\end{array}$ \\
\hline & $\begin{array}{l}\text { Stockwell } \\
2012^{3}\end{array}$ & Canada & $\begin{array}{l}\text { Cross-sectional } \\
\text { versus time } \\
\text { series analysis } \\
\text { of ecological } \\
\text { data }\end{array}$ & $\begin{array}{l}\text { Population of } \\
\text { Saskatchewan }\end{array}$ & $\begin{array}{l}\text { Actual minimum price } \\
\text { increased over a } 7 \text {-year } \\
\text { period. Study modelled a } \\
10 \% \text { increase in the average } \\
\text { minimum price of all } \\
\text { alcoholic beverages adjusted } \\
\text { by monthly Consumer Price } \\
\text { Index }\end{array}$ & $\begin{array}{l}\text { Alcohol } \\
\text { consumption } \\
\text { (measured by sales) }\end{array}$ & Yes & $\begin{array}{l}\text { Not } \\
\text { stated }\end{array}$ & Strong & $\begin{array}{l}\text { SA, CON, TE, } \\
\text { DR, CO, EX }\end{array}$ \\
\hline & $\begin{array}{l}\text { Stockwell } \\
2013^{21}\end{array}$ & Canada & $\begin{array}{l}\text { Cross-sectional } \\
\text { versus time } \\
\text { series analysis } \\
\text { of ecological } \\
\text { data }\end{array}$ & $\begin{array}{l}\text { Populations of } 89 \\
\text { geographic areas in } \\
\text { British Columbia }\end{array}$ & $\begin{array}{l}\text { Actual minimum price } \\
\text { increased over a } 20 \text {-year } \\
\text { period. Study modelled } 10 \% \\
\text { increase in the average } \\
\text { minimum price of all } \\
\text { alcoholic beverages adjusted } \\
\text { by monthly Consumer Price } \\
\text { Index }\end{array}$ & $\begin{array}{l}\text { Alcohol-attributable } \\
\text { hospital admissions }\end{array}$ & Yes & $\begin{array}{l}\text { Not } \\
\text { stated }\end{array}$ & Strong & $\begin{array}{l}\text { SA, CON, TE, } \\
\text { DR, PL, CO, } \\
\text { EX }\end{array}$ \\
\hline & $\begin{array}{l}\text { Treisman } \\
2010^{22}\end{array}$ & Russia & $\begin{array}{l}\text { Secondary } \\
\text { analysis of } \\
\text { historical data } \\
\text { with focus on } \\
\text { price changes } \\
\text { 1990-1994 }\end{array}$ & Population of Russia & $\begin{array}{l}\text { Price liberalisation of vodka } \\
\text { in early } 1990 \text { s-in } 1993 \text {, real } \\
\text { price of vodka was around } \\
25 \% \text { of that in } 1990\end{array}$ & Mortality & Yes & $\begin{array}{l}\text { Not } \\
\text { stated }\end{array}$ & Strong & $\begin{array}{l}\text { SA, CON, TE, } \\
\text { PL, CO, EX }\end{array}$ \\
\hline
\end{tabular}




\begin{tabular}{|c|c|c|c|c|c|c|c|c|c|c|}
\hline \multirow[b]{2}{*}{ Study type } & \multirow[b]{2}{*}{$\begin{array}{l}\text { First author } \\
\text { and year } \\
\text { published }\end{array}$} & \multicolumn{5}{|c|}{ Study characteristics } & \multicolumn{3}{|c|}{ Study assessment } & \multirow[b]{2}{*}{$\begin{array}{l}\text { Bradford Hill } \\
\text { criteria met }\end{array}$} \\
\hline & & Country & Study design & $\begin{array}{l}\text { Population or } \\
\text { participants }\end{array}$ & $\begin{array}{l}\text { Pricing intervention } \\
\text { studied }\end{array}$ & Outcomes studied & $\begin{array}{l}\text { Peer } \\
\text { reviewed }\end{array}$ & $\begin{array}{l}\text { Conflict } \\
\text { of } \\
\text { interest }\end{array}$ & $\begin{array}{l}\text { Quality } \\
\text { rating }\end{array}$ & \\
\hline & Wald $1984^{23}$ & Poland & $\begin{array}{l}\text { Analysis of } \\
\text { routine data } \\
1970-1981\end{array}$ & Population of Poland & $\begin{array}{l}\text { Poor harvest led to high } \\
\text { prices, rationing and illegal } \\
\text { sales }\end{array}$ & $\begin{array}{l}\text { Alcohol } \\
\text { consumption and } \\
\text { alcohol-related } \\
\text { hospital admissions }\end{array}$ & Yes & $\begin{array}{l}\text { Not } \\
\text { stated }\end{array}$ & Weak & $\begin{array}{l}\text { CON, TE, PL, } \\
\text { CO, EX }\end{array}$ \\
\hline & Zhao $2013^{24}$ & Canada & $\begin{array}{l}\text { Cross-sectional } \\
\text { versus time } \\
\text { series analysis } \\
\text { of ecological } \\
\text { data }\end{array}$ & $\begin{array}{l}\text { Populations of } 16 \\
\text { health service } \\
\text { delivery areas in } \\
\text { British Columbia, } \\
\text { Canada }\end{array}$ & $\begin{array}{l}\text { Actual minimum price } \\
\text { increased over a } 20 \text {-year } \\
\text { period. Study modelled } 10 \% \\
\text { increase in the average } \\
\text { minimum price of all } \\
\text { alcoholic beverages adjusted } \\
\text { by monthly Consumer Price } \\
\text { Index. Also looked at outlet } \\
\text { density }\end{array}$ & $\begin{array}{l}\text { Acute, chronic and } \\
\text { wholly } \\
\text { alcohol-attributable } \\
\text { mortality }\end{array}$ & Yes & None & Strong & $\begin{array}{l}\text { SA, CON, TE, } \\
\text { DR, PL, CO, } \\
\text { EX }\end{array}$ \\
\hline \multirow[t]{5}{*}{$\begin{array}{l}\text { Modelling } \\
\text { studies }\end{array}$} & $\begin{array}{l}\text { Brennan } \\
2014^{25}\end{array}$ & England & $\begin{array}{l}\text { Modelling study } \\
\text { using SAPM }\end{array}$ & $\begin{array}{l}\text { The UK national } \\
\text { surveys of general } \\
\text { population } \\
\text { (subgroups of } \\
\text { moderate, harmful, } \\
\text { hazardous) }\end{array}$ & $\begin{array}{l}\text { MUP of } £ 0.40, £ 0.45 \text { and } \\
£ 0.50 . \text { Ban on below cost } \\
\text { selling }\end{array}$ & $\begin{array}{l}\text { Alcohol } \\
\text { consumption, } \\
\text { consumer spending, } \\
47 \text { health harms, } \\
\text { QALYs }\end{array}$ & Yes & None & Strong & $\begin{array}{l}\text { CON, SP, DR, } \\
\text { PL, CO }\end{array}$ \\
\hline & $\begin{array}{l}\text { Holmes } \\
2014^{26}\end{array}$ & England & $\begin{array}{l}\text { Modelling study } \\
\text { using SAPM }\end{array}$ & $\begin{array}{l}\text { The UK national } \\
\text { surveys of general } \\
\text { population } \\
\text { (subgroups of } \\
\text { moderate, harmful, } \\
\text { hazardous) }\end{array}$ & MUP of $45 p$ & $\begin{array}{l}\text { Alcohol } \\
\text { consumption, } \\
\text { consumer spending, } \\
47 \text { health harms, } \\
\text { QALYs }\end{array}$ & Yes & None & Strong & $\begin{array}{l}\text { CON, SP, PL, } \\
\text { CO }\end{array}$ \\
\hline & Meier $2009^{27}$ & The UK & $\begin{array}{l}\text { Modelling study } \\
\text { using SAPM }\end{array}$ & $\begin{array}{l}\text { The UK national } \\
\text { surveys of general } \\
\text { population } \\
\text { (subgroups of } \\
\text { moderate, harmful, } \\
\text { hazardous) }\end{array}$ & $\begin{array}{l}\text { Ten pricing policy options, } \\
\text { including different levels of } \\
\text { MUP (of } 33 \text { analysed) }\end{array}$ & $\begin{array}{l}\text { Alcohol } \\
\text { consumption, } \\
\text { consumer spending, } \\
47 \text { health harms, } \\
\text { crime, employment }\end{array}$ & Yes & None & Strong & $\begin{array}{l}\text { CON, SP, DR, } \\
\text { PL, CO }\end{array}$ \\
\hline & Meier $2016^{28}$ & England & $\begin{array}{l}\text { Modelling study } \\
\text { using SAPM }\end{array}$ & $\begin{array}{l}\text { The UK national } \\
\text { surveys of general } \\
\text { population } \\
\text { (subgroups of } \\
\text { moderate, increasing } \\
\text { risk, heavy) }\end{array}$ & $\begin{array}{l}\text { MUP of } £ 0.50 \text { compared with } \\
\text { three alcohol taxation } \\
\text { interventions }\end{array}$ & $\begin{array}{l}\text { Alcohol } \\
\text { consumption in } \\
\text { different income and } \\
\text { socioeconomic } \\
\text { groups }\end{array}$ & Yes & None & Strong & $\begin{array}{l}\text { CON, SP, PL, } \\
\text { CO }\end{array}$ \\
\hline & $\begin{array}{l}\text { Purshouse } \\
2010^{29}\end{array}$ & England & $\begin{array}{l}\text { Modelling study } \\
\text { using SAPM }\end{array}$ & $\begin{array}{l}\text { The UK national } \\
\text { surveys of general } \\
\text { population } \\
\text { (subgroups of } \\
\text { moderate, harmful, } \\
\text { hazardous) }\end{array}$ & $\begin{array}{l}18 \text { different pricing policies } \\
\text { (including MUP) }\end{array}$ & $\begin{array}{l}\text { Alcohol } \\
\text { consumption, } \\
\text { consumer spending, } \\
47 \text { health harms, } \\
\text { QALYs }\end{array}$ & Yes & None & Strong & $\begin{array}{l}\text { CON, SP, DR, } \\
\text { PL, CO }\end{array}$ \\
\hline
\end{tabular}




\begin{tabular}{|c|c|c|c|c|c|c|c|c|c|c|}
\hline \multirow[b]{2}{*}{ Study type } & \multirow[b]{2}{*}{$\begin{array}{l}\text { First author } \\
\text { and year } \\
\text { published }\end{array}$} & \multicolumn{5}{|c|}{ Study characteristics } & \multicolumn{3}{|c|}{ Study assessment } & \multirow[b]{2}{*}{$\begin{array}{l}\text { Bradford Hill } \\
\text { criteria met }\end{array}$} \\
\hline & & Country & Study design & $\begin{array}{l}\text { Population or } \\
\text { participants }\end{array}$ & $\begin{array}{l}\text { Pricing intervention } \\
\text { studied }\end{array}$ & Outcomes studied & $\begin{array}{l}\text { Peer } \\
\text { reviewed }\end{array}$ & $\begin{array}{l}\text { Conflict } \\
\text { of } \\
\text { interest }\end{array}$ & $\begin{array}{l}\text { Quality } \\
\text { rating }\end{array}$ & \\
\hline \multirow{10}{*}{$\begin{array}{l}\text { Cross- } \\
\text { sectional } \\
\text { studies }\end{array}$} & $\begin{array}{l}\text { Sharma } \\
2016^{30}\end{array}$ & Australia & $\begin{array}{l}\text { Counterfactual } \\
\text { analysis }\end{array}$ & $\begin{array}{l}\text { Representative } \\
\text { sample of } \\
\text { households ( } n=884 \text { ) } \\
\text { completing 12-month } \\
\text { Homescan shopping } \\
\text { survey }\end{array}$ & MUP of $A \$ 2$ & $\begin{array}{l}\text { Alcohol purchasing } \\
\text { and consumption }\end{array}$ & Yes & None & Strong & $\begin{array}{l}\text { CON, SP, PL, } \\
\text { CO }\end{array}$ \\
\hline & $\begin{array}{l}\text { Vandenberg } \\
2016^{31}\end{array}$ & Australia & Modelling study & $\begin{array}{l}\text { Representative } \\
\text { sample of } \\
\text { households ( } n=885 \text { ) } \\
\text { completing } \\
\text { Homescan shopping } \\
\text { survey }\end{array}$ & $\begin{array}{l}\text { MUP of } A \$ 1 \text { compared with } \\
\text { a specific alcohol tax }\end{array}$ & $\begin{array}{l}\text { Alcohol purchasing } \\
\text { and consumption }\end{array}$ & Yes & None & Strong & $\begin{array}{l}\text { CON, SP, PL, } \\
\text { CO }\end{array}$ \\
\hline & Black $2011^{32}$ & Scotland & $\begin{array}{l}\text { Cross-sectional } \\
\text { survey }\end{array}$ & $\begin{array}{l}377 \text { hospital patients } \\
\text { with serious alcohol } \\
\text { problems }\end{array}$ & $\begin{array}{l}\text { The UK alcohol units } \\
\text { purchased below proposed } \\
\text { MUP of } £ 0.40 p / £ 0.50 p\end{array}$ & $\begin{array}{l}\text { Alcohol } \\
\text { consumption }\end{array}$ & Yes & None & Moderate & $\begin{array}{l}\text { SA CON, DR, } \\
\text { PL, CO }\end{array}$ \\
\hline & $\begin{array}{l}\text { Callinan } \\
2015^{33}\end{array}$ & Australia & $\begin{array}{l}\text { Cross-sectional } \\
\text { survey }\end{array}$ & $\begin{array}{l}\text { Drinkers } 18+ \\
\text { participating in } \\
\text { Australian } \\
\text { International Alcohol } \\
\text { Control study } \\
(n=1681)\end{array}$ & $\begin{array}{l}\text { Australian standard drinks } \\
\text { purchased below proposed } \\
\text { minimum prices of } A \$ 0.80 / A \\
\$ 1.00 / A \$ 1.25\end{array}$ & $\begin{array}{l}\text { Alcohol } \\
\text { consumption }\end{array}$ & Yes & $\begin{array}{l}\text { Not } \\
\text { stated }\end{array}$ & Moderate & $\begin{array}{l}\text { SA, CON, DR, } \\
\text { PL, CO }\end{array}$ \\
\hline & $\begin{array}{l}\text { Cousins } \\
2016^{34}\end{array}$ & Ireland & $\begin{array}{l}\text { Cross-sectional } \\
\text { survey }\end{array}$ & $\begin{array}{l}3187 \text { adults in } 2013 \\
\text { National Alcohol } \\
\text { Diary Survey }\end{array}$ & $\begin{array}{l}\text { Alcohol units purchased } \\
\text { below proposed minimum } \\
\text { price of } € 1.00\end{array}$ & AUDIT-C score & Yes & None & Strong & $\begin{array}{l}\text { SA, CON, PL, } \\
\text { CO }\end{array}$ \\
\hline & $\begin{array}{l}\text { Crawford } \\
2012^{35}\end{array}$ & England & $\begin{array}{l}\text { Cross-sectional } \\
\text { survey }\end{array}$ & $\begin{array}{l}515 \text { members of the } \\
\text { public }\end{array}$ & $\begin{array}{l}\text { The UK alcohol units } \\
\text { purchased below proposed } \\
\text { MUP of } £ 0.50\end{array}$ & AUDIT score & Yes & None & Moderate & $\begin{array}{l}\text { SA, CON, PL, } \\
\text { CO }\end{array}$ \\
\hline & $\begin{array}{l}\text { Falkner } \\
2015^{36}\end{array}$ & $\begin{array}{l}\text { New } \\
\text { Zealand }\end{array}$ & $\begin{array}{l}\text { Cross-sectional } \\
\text { survey }\end{array}$ & $\begin{array}{l}115 \text { adults } \\
\text { undergoing alcohol } \\
\text { detoxification }\end{array}$ & $\begin{array}{l}\text { New Zealand standard } \\
\text { drinks purchased below } \\
\text { proposed minimum prices of } \\
\mathrm{NZ} \$ 1.00 / \mathrm{NZ} \$ 1.10 / \mathrm{NZ} \$ 1.20\end{array}$ & $\begin{array}{l}\text { Alcohol } \\
\text { consumption }\end{array}$ & Yes & No & Moderate & $\begin{array}{l}\text { SA, CON, PL, } \\
\text { CO }\end{array}$ \\
\hline & $\begin{array}{l}\text { Forsyth } \\
2014^{37}\end{array}$ & Scotland & $\begin{array}{l}\text { Cross-sectional } \\
\text { survey }\end{array}$ & $\begin{array}{l}\text { Shopkeepers of } 144 \\
\text { off licences in } \\
\text { Glasgow }\end{array}$ & MUP of $£ 0.50$ & $\begin{array}{l}\text { Products affected } \\
\text { and hospital } \\
\text { admissions }\end{array}$ & Yes & None & Weak & $\begin{array}{l}\text { CON, PL } \\
\text { (weakly), CO }\end{array}$ \\
\hline & $\begin{array}{l}\text { Ludbrook } \\
2012^{38}\end{array}$ & The UK & $\begin{array}{l}\text { Cross-sectional } \\
\text { survey }\end{array}$ & $\begin{array}{l}\text { Expenditure and } \\
\text { Food Survey data } \\
\text { from 20062008 } \\
(n=18624)\end{array}$ & $\begin{array}{l}\text { Purchasers of alcohol }< \\
£ 0.45 \text { per unit }\end{array}$ & $\begin{array}{l}\text { Income of } \\
\text { purchasers of cheap } \\
\text { alcohol }\end{array}$ & Yes & $\begin{array}{l}\text { Not } \\
\text { stated }\end{array}$ & Moderate & $\begin{array}{l}\text { SA, CON, PL, } \\
\text { CO }\end{array}$ \\
\hline & $\begin{array}{l}\text { Sharma } \\
2014^{39}\end{array}$ & Australia & $\begin{array}{l}\text { Cross-sectional } \\
\text { survey }\end{array}$ & $\begin{array}{l}\text { Representative } \\
\text { sample of } \\
\text { households ( } n=885 \text { ) } \\
\text { completing shopping } \\
\text { survey }\end{array}$ & MUP of $A \$ 1$ and taxation & $\begin{array}{l}\text { Alcohol } \\
\text { consumption } \\
\text { (measured by } \\
\text { projected sales) }\end{array}$ & Yes & None & Moderate & $\begin{array}{l}\text { SA, CON, DR, } \\
\text { PL, CO }\end{array}$ \\
\hline
\end{tabular}


Table 2 Continued

\begin{tabular}{|c|c|c|c|c|c|c|c|c|c|c|}
\hline \multirow[b]{2}{*}{ Study type } & \multirow[b]{2}{*}{$\begin{array}{l}\text { First author } \\
\text { and year } \\
\text { published }\end{array}$} & \multicolumn{5}{|c|}{ Study characteristics } & \multicolumn{3}{|c|}{ Study assessment } & \multirow[b]{2}{*}{$\begin{array}{l}\text { Bradford Hill } \\
\text { criteria met }\end{array}$} \\
\hline & & Country & Study design & $\begin{array}{l}\text { Population or } \\
\text { participants }\end{array}$ & $\begin{array}{l}\text { Pricing intervention } \\
\text { studied }\end{array}$ & Outcomes studied & $\begin{array}{l}\text { Peer } \\
\text { reviewed }\end{array}$ & $\begin{array}{l}\text { Conflict } \\
\text { of } \\
\text { interest }\end{array}$ & $\begin{array}{l}\text { Quality } \\
\text { rating }\end{array}$ & \\
\hline & $\begin{array}{l}\text { Sheron } \\
2014^{40}\end{array}$ & The UK & $\begin{array}{l}\text { Cross-sectional } \\
\text { survey }\end{array}$ & $\begin{array}{l}\text { Adult patients in a } \\
\text { liver unit of a hospital } \\
(n=204)\end{array}$ & $\begin{array}{l}\text { The UK alcohol units } \\
\text { purchased below £0.50 }\end{array}$ & $\begin{array}{l}\text { Alcohol } \\
\text { consumption }\end{array}$ & Yes & $\begin{array}{l}\text { Not } \\
\text { stated }\end{array}$ & Moderate & $\begin{array}{l}\text { SA, CON, DR, } \\
\text { PL, CO }\end{array}$ \\
\hline $\begin{array}{l}\text { Intervention } \\
\text { studies }\end{array}$ & Babor $1978^{41}$ & The USA & $\begin{array}{l}\text { Trial (not } \\
\text { randomised) }\end{array}$ & $\begin{array}{l}34 \text { male volunteers in } \\
\text { live-in research } \\
\text { facility }\end{array}$ & $\begin{array}{l}\text { 'Happy hour' with a reduction } \\
\text { in set price of alcohol for one } \\
\text { group of participants }\end{array}$ & $\begin{array}{l}\text { Alcohol } \\
\text { consumption }\end{array}$ & Yes & $\begin{array}{l}\text { Not } \\
\text { stated }\end{array}$ & Weak & $\begin{array}{l}\text { SA, CON, SP, } \\
\text { TE, CO, EX }\end{array}$ \\
\hline $\begin{array}{l}\text { Qualitative } \\
\text { studies }\end{array}$ & $\begin{array}{l}\text { Seaman } \\
2013^{42}\end{array}$ & Scotland & $\begin{array}{l}\text { Qualitative } \\
\text { study }\end{array}$ & $\begin{array}{l}130 \text { participants aged } \\
16-30\end{array}$ & $\begin{array}{l}\text { Hypothetical minimum price } \\
\text { increases }\end{array}$ & $\begin{array}{l}\text { Alcohol } \\
\text { consumption and } \\
\text { substitution with } \\
\text { other substances }\end{array}$ & Yes & None & Moderate & $\mathrm{CON}, \mathrm{CO}$ \\
\hline \multirow[t]{2}{*}{$\begin{array}{l}\text { Systematic } \\
\text { reviews }\end{array}$} & $\begin{array}{l}\text { Wagenaar } \\
2009^{43}\end{array}$ & Worldwide & $\begin{array}{l}\text { Systematic } \\
\text { review and } \\
\text { meta-analysis }\end{array}$ & $\begin{array}{l}\text { Studies tended to } \\
\text { cover general } \\
\text { population }\end{array}$ & $\begin{array}{l}\text { Alcohol price and taxation } \\
\text { interventions studied } \\
\text { together }\end{array}$ & $\begin{array}{l}\text { Alcohol } \\
\text { consumption } \\
\text { (measured by } \\
\text { alcohol sales or } \\
\text { self-reported } \\
\text { consumption) }\end{array}$ & Yes & None & Strong & AN \\
\hline & $\begin{array}{l}\text { Wagenaar } \\
2010^{44}\end{array}$ & Worldwide & $\begin{array}{l}\text { Systematic } \\
\text { review and } \\
\text { meta-analysis }\end{array}$ & $\begin{array}{l}\text { Studies tended to } \\
\text { cover general } \\
\text { population }\end{array}$ & $\begin{array}{l}\text { Alcohol price and taxation } \\
\text { interventions studied } \\
\text { together }\end{array}$ & $\begin{array}{l}\text { Alcohol-related } \\
\text { morbidity (disease, } \\
\text { injury, suicide, traffic } \\
\text { crashes, sexually } \\
\text { transmitted } \\
\text { diseases, other } \\
\text { drug use, crime and } \\
\text { misbehaviour) and } \\
\text { mortality }\end{array}$ & Yes & $\begin{array}{l}\text { Not } \\
\text { stated }\end{array}$ & Strong & AN \\
\hline
\end{tabular}


Table 3 Studies published in the grey literature included in Bradford Hill criteria assessment

\begin{tabular}{|c|c|c|c|c|c|c|c|c|c|}
\hline \multirow{2}{*}{$\begin{array}{l}\text { Author and } \\
\text { year } \\
\text { published }\end{array}$} & \multicolumn{5}{|c|}{ Study characteristics } & \multicolumn{3}{|c|}{ Study assessment } & \multirow{2}{*}{$\begin{array}{l}\text { Bradford } \\
\text { Hill criteria } \\
\text { met }\end{array}$} \\
\hline & Country & Study design & $\begin{array}{l}\text { Population or } \\
\text { participants }\end{array}$ & $\begin{array}{l}\text { Pricing intervention } \\
\text { studied }\end{array}$ & Outcomes studied & $\begin{array}{l}\text { Peer } \\
\text { reviewed }\end{array}$ & $\begin{array}{l}\text { Conflict } \\
\text { of interest }\end{array}$ & $\begin{array}{l}\text { Quality } \\
\text { rating }\end{array}$ & \\
\hline Angus $2016^{45}$ & Scotland & $\begin{array}{l}\text { Modelling } \\
\text { study using } \\
\text { SAPM }\end{array}$ & $\begin{array}{l}\text { Scottish general } \\
\text { population survey } \\
\text { (subgroups of } \\
\text { moderate, harmful, } \\
\text { hazardous) }\end{array}$ & $\begin{array}{l}\text { MUP of } 30 p, 40 p, 50 p \\
60 p \text { and } 70 p \text {, compared } \\
\text { with taxation interventions }\end{array}$ & $\begin{array}{l}\text { Alcohol consumption, } \\
\text { consumer spending, } \\
\text { exchequer and retail } \\
\text { revenue, } 47 \text { health } \\
\text { harms }\end{array}$ & Not stated & None & Strong & $\begin{array}{l}\text { CON, SP, } \\
\text { DR, PL, } \\
\text { CO }\end{array}$ \\
\hline Booth $2008^{46}$ & Worldwide & $\begin{array}{l}\text { Review of } \\
\text { reviews and } \\
\text { systematic } \\
\text { review }\end{array}$ & $\begin{array}{l}\text { Studies tended to } \\
\text { cover general } \\
\text { population }\end{array}$ & $\begin{array}{l}\text { Various minimum unit } \\
\text { prices and taxation } \\
\text { interventions }\end{array}$ & $\begin{array}{l}\text { Alcohol consumption } \\
\text { and various measures } \\
\text { of alcohol harm }\end{array}$ & Yes & None & Strong & AN \\
\hline $\begin{array}{l}\text { Brennan } \\
2008^{47}\end{array}$ & England & $\begin{array}{l}\text { Modelling } \\
\text { study using } \\
\text { SAPM }\end{array}$ & Adults in England & $\begin{array}{l}\text { General price increases. } \\
\text { MUP of } £ 0.20, £ 0.25 \text {, } \\
£ 0.30, £ 0.35, £ 0.40 \text {, } \\
£ 0.45, £ 0.50, £ 0.60 \text { and } \\
£ 0.70 \text {. Restrictions on } \\
\text { off-trade price promotions. }\end{array}$ & $\begin{array}{l}\text { Alcohol consumption, } \\
\text { consumer spending, } \\
\text { sales duty and VAT, } \\
47 \text { health harms, } \\
\text { crime and employment }\end{array}$ & Not stated & None & Strong & $\begin{array}{l}\text { CON, SP, } \\
\text { DR, PL, } \\
\text { CO }\end{array}$ \\
\hline $\begin{array}{l}\text { Hill McManus } \\
2012^{48}\end{array}$ & Canada & $\begin{array}{l}\text { Modelling } \\
\text { study using } \\
\text { SAPM }\end{array}$ & $\begin{array}{l}\text { Adults in two } \\
\text { Canadian } \\
\text { provinces (Ontario } \\
\text { and British } \\
\text { Columbia) }\end{array}$ & MUP of $C \$ 1.50$ & $\begin{array}{l}\text { Alcohol consumption, } \\
\text { consumer spending, } \\
\text { hospital admissions, } \\
\text { mortality, crime }\end{array}$ & No & None & Strong & $\begin{array}{l}\text { CON, SP, } \\
\text { PL, CO }\end{array}$ \\
\hline $\begin{array}{l}\text { Institute for } \\
\text { Fiscal Studies } \\
2010^{49}\end{array}$ & $\begin{array}{l}\text { Great } \\
\text { Britain }\end{array}$ & $\begin{array}{l}\text { Economic } \\
\text { modelling } \\
\text { study using } \\
\text { market } \\
\text { research data }\end{array}$ & $\begin{array}{l}\text { Shopping data } \\
\text { from } 25248 \text { British } \\
\text { households }\end{array}$ & MUP of $£ 0.45$ & Alcohol consumption & Not stated & Not stated & $\begin{array}{l}\text { Not } \\
\text { possible } \\
\text { to rate }\end{array}$ & $\begin{array}{l}\mathrm{CON}, \mathrm{SP} \\
\mathrm{CO}\end{array}$ \\
\hline $\begin{array}{l}\text { Institute for } \\
\text { Fiscal Studies } \\
2013^{50}\end{array}$ & $\begin{array}{l}\text { Great } \\
\text { Britain }\end{array}$ & $\begin{array}{l}\text { Economic } \\
\text { analysis }\end{array}$ & $\begin{array}{l}\text { Population of Great } \\
\text { Britain }\end{array}$ & $\begin{array}{l}\text { MUP of } £ 0.45 \text { and } \\
\text { increased alcohol taxation }\end{array}$ & Alcohol consumption & Not stated & Not stated & $\begin{array}{l}\text { Not } \\
\text { possible } \\
\text { to rate }\end{array}$ & $\begin{array}{l}\text { CON, SP, } \\
\text { CO }\end{array}$ \\
\hline Meng $2010^{51}$ & Scotland & $\begin{array}{l}\text { Modelling } \\
\text { study using } \\
\text { SAPM }\end{array}$ & Adults in Scotland & $\begin{array}{l}\text { MUP of } £ 0.20, £ 0.25 \text {, } \\
£ 0.30, £ 0.35, £ 0.40 \text {, } \\
£ 0.45, £ 0.50, £ 0.60 \text { and } \\
£ 0.70 \text {. Restrictions on } \\
\text { off-trade price promotions. }\end{array}$ & $\begin{array}{l}\text { Alcohol consumption, } \\
\text { consumer spending, } \\
47 \text { health harms, } \\
\text { crime, employment }\end{array}$ & Not stated & None & Strong & $\begin{array}{l}\text { CON, SP, } \\
\text { DR, PL, } \\
\text { CO }\end{array}$ \\
\hline
\end{tabular}


comes from research following the introduction of MUP in Canada, where minimum price increases preceded reductions in alcohol consumption, ${ }^{2}{ }^{3}$ alcohol-attributable hospital admissions ${ }^{21}$ and alcohol-related mortality. ${ }^{24}$ Studies where price changes preceded the expected changes in alcohol consumption or harm have also been conducted in Russia, ${ }^{19} 22$ Poland $^{23}$ and Finland. ${ }^{20}$ Overall, there is very strong support for the temporality criterion.

\section{Dose-response/biological gradient}

This criterion is supported if different price levels have been found to have differing effects on consumption or harm. Many of the studies using the Sheffield Alcohol Policy Model explore the impact of a range of potential MUP options, ${ }^{25} 27294551$ and these consistently suggest that the higher the MUP the greater the reductions in alcohol consumption or alcohol-related harms. The Canadian studies of minimum pricing lend further support for this criterion because the analysis presents the effect on consumption or harm of a modelled $1 \%$ increase in price, meaning dose response can be inferred. $^{23}{ }^{21}{ }^{24}$ Dose response is supported to a lesser extent by evidence from cross-sectional studies that heavier drinkers are more likely to pay less than a proposed MUP. ${ }^{32-34} 3940$ Overall, there is strong support for the dose-response criterion, although the relationship is difficult to quantify.

\section{Plausibility}

This criterion refers to whether there is evidence that alcohol price can be used as an economic mechanism to influence consumption at a population level, and whether heavy drinkers tend to purchase cheaper alcohol. There is evidence from 21/26 research studies and $4 / 7$ studies in the grey literature that the price of alcohol is inversely related to alcohol-related morbidity, hospital admissions or mortality. Moreover, there is also evidence from numerous cross-sectional studies in the UK, Ireland and Australia ${ }^{32-36} 38-40$ and one trial from the $\mathrm{USA}^{41}$ that heavier drinking was significantly associated with purchasing alcohol below specified prices, further suggesting that economic mechanisms such as minimum pricing would particularly affect the heaviest drinkers. This provides strong support for the plausibility criterion.

\section{Coherence}

This criterion refers to whether studies from different disciplines have had complementary findings and whether these fit or 'hang' well together. It is different to consistency, which is more concerned with reproducibility of findings. The findings of the majority of studies supported the coherence criterion in that they suggest

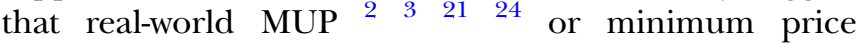
increases ${ }^{19} 2023$ led to reductions in alcohol consumption and alcohol-related harm and cross-sectional surveys find that it is the heavier drinkers that are drinking the cheapest alcohol. ${ }^{32} 40$ The modelling studies which use survey data in turn suggest heavier drinkers will be most affected by MUP. ${ }^{26}$ Overall, the evidence base provides strong support for this criterion.

\section{Experiment}

We have not identified any RCTs of minimum pricing or price-based interventions to reduce alcohol consumption. There is a small (and not randomised) trial from the $1970 \mathrm{~s}^{41}$ which found participants living in controlled conditions and offered a daily 'happy hour' discount drank significantly more alcohol than those who were not offered the discount. There is, however, substantial evidence in support of the experiment criterion from time series analyses or natural experiments, for example, where minimum pricing was introduced in Canada ${ }^{3} 2124$ and where prices fluctuated in the late 1980s and early 1990s in Russia, ${ }^{19}{ }^{22}$ and to a lesser extent in Finland, where minimum price increases were associated with reduced mortality only among men with a basic education. ${ }^{20}$ These studies provide tentative support for the experiment criterion.

\section{Analogy}

To address the analogy criterion, areas related to minimum alcohol pricing must be considered. There is evidence from literature on the affordability of alcohol ${ }^{52}$ that consumption and harm are very responsive to the affordability of alcohol. Large systematic reviews have investigated the price elasticity of demand for alcohol, ${ }^{53}$ and have found that higher alcohol pricing and taxation (considered together) are associated with reductions in alcohol consumption, alcohol-related morbidity and mortality. ${ }^{43} 4446$ Overall, the support for the analogy criterion is very strong, although Bradford Hill describes this as the weakest evidence for causality.

\section{DISCUSSION}

We assessed 26 research studies and two systematic reviews, plus a further seven studies from the grey literature in this review of the evidence for priced-based interventions-such as MUP-to reduce alcohol consumption and alcohol-related harm. All nine of the Bradford Hill criteria for causality were met and the vast majority of studies offered support for price-based alcohol policy interventions. However, the evidence for two of the criteria, although present, was not as strong as it was for the other criteria. These criteria were strength of the association (criterion 1) and experiment (criterion 8), and according to Bradford Hill, these are the two criteria that can provide the strongest evidence for causality. Therefore, although all of the criteria were supported, we conclude that it is highly probable, but not definite, that introducing MUP for alcohol would reduce alcohol consumption and alcohol-related harms. It is also of note that different types of study tended to satisfy different Bradford Hill criteria, and that different 
study designs also produced evidence of the effectiveness of minimum pricing in relation to different outcomes. This is summarised in figure 2. This underlines the importance of including a variety of study designs in this review.

Strengths of this study are that this is the first to have systematically reviewed the literature relevant specifically to alcohol minimum pricing policies. We had broad inclusion criteria with regards to study design, price intervention and outcome measure, allowing for a comprehensive review of the evidence base. Application of the Bradford Hill criteria as part of a narrative systematic literature review is a useful and emergent technique for identifying causality: a PubMed search for systematic reviews with 'Bradford Hill' mentioned in the title or abstract yielded 28 results, $90 \%$ of which were published in the last 5 years. The limitations of this systematic review relate mainly to the broad range of studies included. It was not possible to conduct any kind of meta-analysis and therefore we do not present a pooled estimate for the likely effect of MUP on certain outcomes. The exact effect of any MUP would be influenced by a range of factors, including: the minimum price level chosen, how broadly it is applied, how strongly it is enforced and contextual factors such as affordability (in the UK, alcohol was 54\% more affordable in 2014 than it was in $1980^{16}$ ), other governmental regulations and the price-level pre-MUP. Occasionally, minimum pricing has been implemented as part of a range of measures, ${ }^{19}$ and these studies were considered alongside studies where MUP was implemented in isolation. This emphasises the importance of the specificity criterion.

There were also challenges with the quality appraisal. The EPHPP quality assessment tool was used to assess quantitative studies and the majority of studies were rated as strong or moderate. However, it was not possible to appraise two of the studies from the grey literature using this tool, and there were some challenges assessing the econometric modelling studies against this framework. However, overall we think that our quality appraisal across the different studies is broadly comparable. It should also be noted that although a number of studies were rated as 'strong', this is in relation to their respective study designs and does not reflect the position of the study type in the hierarchy of evidence framework.

This is the first systematic review that has addressed the effectiveness of minimum alcohol price interventions such as MUP using the Bradford Hill criteria. It was beyond the scope of this review to study the impact of generalised increases in alcohol prices (as opposed to minimum prices). However, where such studies have been carried out, a minimum price or floor price has been recommended, for example, in Gruenewald's 2006 study in Sweden which found that the lowest quality (the cheapest) alcohol has the highest price elasticity. ${ }^{54}$ Previous systematic reviews of alcohol price and consumption $^{43}$ and alcohol-related harm ${ }^{44}$ have tended to consider the effect of price increases and increased taxation together. These reviews found significant effects on consumption and morbidity and mortality. Although price regulation and taxation are closely related policy options, evidence from surveys ${ }^{55}$ and modelling studies ${ }^{45}$ suggests that the effects of each are different, although it is known that the majority of tax increases are passed on as increased prices for consumers. ${ }^{43}{ }^{56}$ It was beyond the scope of this review to discuss whether MUP is regressive in detail, but as it only affects the prices of the cheapest drinks, which are usually consumed by the heaviest drinkers, MUP is likely to narrow health inequalities. $^{28} \quad 31$ A recent rapid evidence review
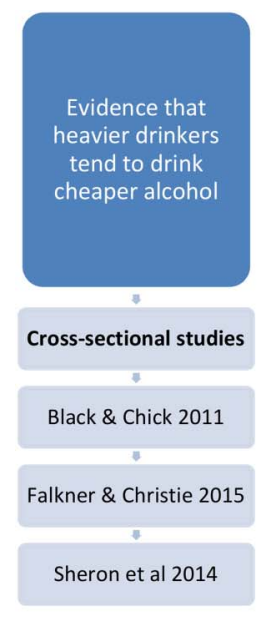
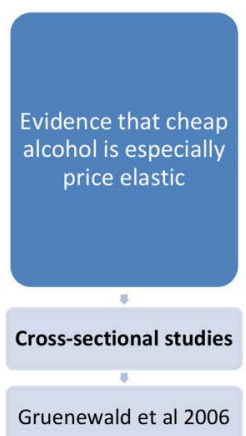

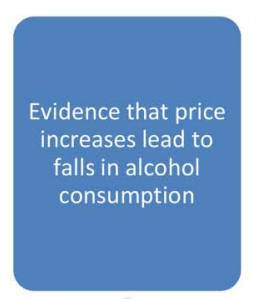

Systematic reviews

Wagenaar et al 2009

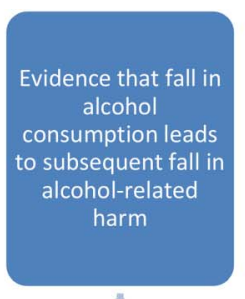

Systematic reviews

Wagenaar et al 2010
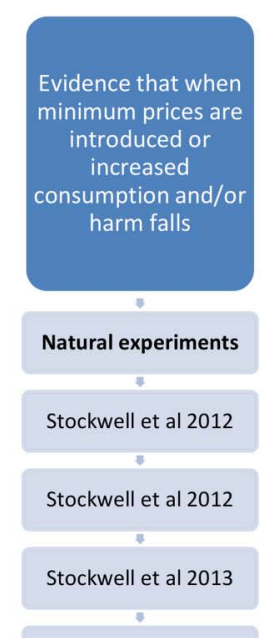

Zhao et al 2013

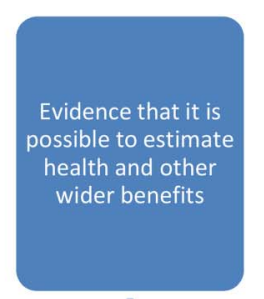

Modelling studies

Brennan et al 2014

Holmes et al 2014

Meier et al 2009

Fure 2 This model shows that different study types tended to produce evidence of effectiveness of minimum pricing in relation to different outcomes. Studies cited in the figure are key examples of the literature in that area and do not represent an exhaustive list. 
published in The Lancet examined alcohol control policies in England and recommended a combination of MUP and tax increases to reduce alcohol harm and increase government revenue, rather than either in isolation. ${ }^{57}$ It is also important to highlight that a considerable proportion of included studies were produced by a small number of research teams. Also, with regards to the econometric modelling studies, uncertainty in estimates or forecasts is often poorly communicated outside of the academic literature. The overall risk of bias in the included studies was minimised by excluding studies with a conflict of interest (either for or against MUP). It was not possible to assess publication bias using an analytical technique such as a funnel plot due to the narrative nature of the review; however, we anticipate that by including grey literature in this review, we have mitigated publication bias as far as reasonably possible.

Overall, the findings of this review lend strong support for policies such as MUP in reducing alcohol consumption and alcohol-related harm, with all nine of the Bradford Hill criteria met, and little by way of counter findings. As it is unlikely to be feasible to conduct RCTs of MUP, the decision whether or not to introduce MUP will not be informed by a systematic review and meta-analysis of RCTs, and therefore, this synthesis of evidence according to the Bradford Hill criteria is of value.

Unanswered questions about the effectiveness of MUP remain; for example, this review has highlighted that support was moderate or tentative for two of the Bradford Hill criteria ('strength of the association' and 'experiment', respectively). There may be opportunities to explore this in countries such as Scotland if MUP is implemented. If Scotland were to implement MUP, then it would be possible to evaluate the validity of the Sheffield Alcohol Policy Model studies conducted using Scottish data. It would also be possible to conduct a longitudinal study to evaluate the effectiveness of MUP in reducing alcohol consumption and alcohol-related morbidity and mortality. The findings of this natural experiment would have relevance elsewhere within and outside the UK.

\section{Twitter Follow Sadie Boniface @sadieboniface}

Acknowledgements We would like to thank Rebecca McDonald for advice on using the Bradford Hill criteria in a systematic review, Dr James Nicholls for advice on study interpretation and Dr Daniel Stahl for statistical advice on some of the included studies.

Contributors SM conceived the idea. SB conducted the initial search. SM and SM contributed to independently reviewing abstracts, hand-searching reference lists, completing data extraction and conducting quality appraisal. All authors contributed to the analysis and interpretation of the results and contributed to writing the manuscript. SB is guarantor.

Funding Open access for this article was funded by King's College London Open Scholarship Fund.

Competing interests SB and SM work at King's College London, which as an institution is listed as a member of the Alcohol Health Alliance. SM has received funding indirectly from UKCTAS, which as an institution is also listed as a member of the Alcohol Health Alliance. None of the authors have any relationship with the Alcohol Health Alliance.
Provenance and peer review Not commissioned; externally peer reviewed.

Data sharing statement No additional data are available.

Open Access This is an Open Access article distributed in accordance with the terms of the Creative Commons Attribution (CC BY 4.0) license, which permits others to distribute, remix, adapt and build upon this work, for commercial use, provided the original work is properly cited. See: http:// creativecommons.org/licenses/by/4.0/

\section{REFERENCES}

1. Anderson P, Chisholm D, Fuhr DC. Effectiveness and cost-effectiveness of policies and programmes to reduce the harm caused by alcohol. Lancet 2009;373:2234-46

2. Stockwell T, Auld MC, Zhao J, et al. Does minimum pricing reduce alcohol consumption? The experience of a Canadian province. Addiction 2012;107:912-20.

3. Stockwell T, Zhao J, Giesbrecht N, et al. The raising of minimum alcohol prices in Saskatchewan, Canada: impacts on consumption and implications for public health. Am J Public Health 2012;102: e103-10.

4. World Health Organization. European status report on alcohol and health 2014. Pricing policies. World Health Organization, 2014. http:// www.euro.who.int/_data/assets/pdf_file/0003/244902/ Pricing-policies pdf

5. Department of Health (Ireland). The Public Health (Alcohol) Bill 2015. 2015 (cited 17 February 2017). http://health.gov.ie/blog/ publications/public-health-alcohol-bill-2015/

6. The Guardian. Minimum alcohol price not in the public interest, says health agency. The Guardian. 1 May 2014 (cited 17 February 2017) https://www.theguardian.com/world/2014/may/01/minimum-alcoholprice-not-in-the-public-interest-says-health-agency

7. New Zealand Ministry of Justice. Alcohol minimum pricing policies. (cited 17 February 2017). https://justice.govt.nz/justice-sector-policy/ key-initiatives/sale-and-supply-of-alcohol/alcohol-minimum-pricingreport/

8. Home Office. Guidance on banning the sale of alcohol below the cost of duty plus VAT. For suppliers of alcohol and enforcement authorities in England and Wales. 2015 (cited 3 June 2016). Available from: https://www.gov.uk/government/uploads/system/ uploads/attachment data/file/415522/HO Guidance on BBCS.pdf

9. Off Licence News. First conviction for selling alcohol "below cost" sees retailer fined. 2016 (cited 3 June 2016). http://www. offlicencenews.co.uk/news/fullstory.php/aid/15750/First_conviction for_selling_alcohol_below_cost_sees_retailer_fined.html

10. Home Office. Duty plus VAT permitted price calculator (2015). 2016 https://www.gov.uk/government/publications/banning-the-sale-ofalcohol-below-the-cost-of-duty-plus-vat-march-2015

11. HM Government. The Government's Alcohol Strategy. 2012 (cited 3 June 2016). https://www.gov.uk/government/uploads/system/ uploads/attachment_data/file/224075/alcohol-strategy.pdf

12. Scottish Government. Minimum unit pricing. (cited 17 February 2017). http://www.gov.scot/Topics/Health/Services/Alcohol/ minimum-pricing

13. Court of Justice of the European Union. Press Release 155/15: the Scottish legislation introducing a minimum price per unit of alcohol is contrary to EU law if less restrictive tax measures can be introduced 2015 (cited 17 February 2017). http://curia.europa.eu/jcms/upload/ docs/application/pdf/2015-12/cp150155en.pdf

14. Judiciary of Scotland. Scotch Whisky Association and others v Lord Advocate and Advocate General for Scotland. (cited 11 July 2016). http://www.scotland-judiciary.org.uk/9/1672/Scotch-WhiskyAssociation-and-others-v-Lord-Advocate-and-Advocate-Generalfor-Scotland

15. BBC News. Bid to appeal against minimum alcohol pricing to Supreme Court. 2016 Nov 18 (cited 17 February 2017) http://www. bbc.co.uk/news/uk-scotland-scotland-politics-38026073

16. Lifestyles Statistics Team, Health and Social Care Information Centre. Statistics on alcohol. England, 2015. 2015 (cited 3 June 2016). http://www.hscic.gov.uk/catalogue/PUB17712/ alc-eng-2015-rep.pdf

17. Cochrane Handbook. 21.4 Assessment of study quality and risk of bias. (cited 2 June 2016). http://handbook.cochrane.org/chapter_21/ 214 assessment of study quality and risk of bias.htm

18. Hill $A \bar{B}$. The environment and disease: association or causation? Proc $R$ Soc Med 1965;58:295-300.

19. Bhattacharya J, Gathmann C, Miller G. The Gorbachev Anti-Alcohol Campaign and Russia's Mortality Crisis. Am Econ J Appl Econ 2013:5:232-60. 
20. Herttua K, Mäkelä P, Martikainen P. Minimum prices for alcohol and educational disparities in alcohol-related mortality. Epidemiology 2015;26:337-43.

21. Stockwell T, Zhao J, Martin G, et al. Minimum alcohol prices and outlet densities in British Columbia, Canada: estimated impacts on alcohol-attributable hospital admissions. Am J Public Health 2013;103:2014-20.

22. Treisman D. Death and prices. Econ Transit 2010;18:281-331.

23. Wald I, Moskalewicz J. Alcohol policy in a crisis situation. Br J Addict 1984;79:331-5.

24. Zhao J, Stockwell T, Martin G, et al. The relationship between minimum alcohol prices, outlet densities and alcohol-attributable deaths in British Columbia, 2002-09. Addiction 2013;108: 1059-69.

25. Brennan A, Meng $Y$, Holmes $J$, et al. Potential benefits of minimum unit pricing for alcohol versus a ban on below cost selling in England 2014: modelling study. BMJ 2014;349:g5452.

26. Holmes J, Meng Y, Meier PS, et al. Effects of minimum unit pricing for alcohol on different income and socioeconomic groups: a modelling study. Lancet 2014;383:1655-64.

27. Meier PS, Purshouse R, Brennan A. Policy options for alcohol price regulation: the importance of modelling population heterogeneity. Addiction 2010;105:383-93.

28. Meier PS, Holmes J, Angus C, et al. Estimated effects of different alcohol taxation and price policies on health inequalities: a mathematical modelling study. PLOS Med 2016;13:e1001963.

29. Purshouse RC, Meier PS, Brennan A, et al. Estimated effect of alcohol pricing policies on health and health economic outcomes in England: an epidemiological model. Lancet 2010;375:1355-64.

30. Sharma A, Etilé $F$, Sinha K. The effect of introducing a minimum price on the distribution of alcohol purchase: a counterfactual analysis. Health Econ 2016;25:1182-200.

31. Vandenberg B, Sharma A. Are alcohol taxation and pricing policies regressive? Product-level effects of a specific tax and a minimum unit price for alcohol. Alcohol Alcohol 2016;51:493-502.

32. Black $\mathrm{H}$, Gill J, Chick J. The price of a drink: levels of consumption and price paid per unit of alcohol by Edinburgh's ill drinkers with a comparison to wider alcohol sales in Scotland. Addiction 2011;106:729-36.

33. Callinan S, Room R, Livingston M, et al. Who purchases low-cost alcohol in Australia? Alcohol Alcohol 2015;50:647-53.

34. Cousins G, Mongan D, Barry J, et al. Potential impact of minimum unit pricing for alcohol in Ireland: evidence from the national alcohol diary survey. Alcohol Alcohol 2016:51:734-40.

35. Crawford MJ, Parry AMH, Weston ARW, et al. Relationship between price paid for off-trade alcohol, alcohol consumption and income in England: a cross-sectional survey. Alcohol Alcohol 2012;47:738-42.

36. Falkner C, Christie G, Zhou L. The effect of alcohol price on dependent drinkers' alcohol consumption. $N Z$ Med $J$ 2015;128:9-17.

37. Forsyth AJM, Ellaway A, Davidson N. How might the alcohol minimum unit pricing (MUP) impact upon local off-sales shops and the communities which they serve? Alcohol Alcohol 2014:49:96-102.

38. Ludbrook A, Petrie D, McKenzie L, et al. Tackling alcohol misuse: purchasing patterns affected by minimum pricing for alcohol. Appl Health Econ Health Policy 2012;10:51-63.

39. Sharma A, Vandenberg B, Hollingsworth B. Minimum pricing of alcohol versus volumetric taxation: which policy will reduce heavy consumption without adversely affecting light and moderate consumers? PLOS ONE 2014;9:e80936.

40. Sheron N, Chilcott F, Matthews L, et al. Impact of minimum price per unit of alcohol on patients with liver disease in the UK. Clin Med 2014;14:396-403.
41. Babor TF, Mendelson JH, Greenberg I, et al. Experimental analysis of the 'happy hour': effects of purchase price on alcohol consumption. Psychopharmacology (Berl) 1978;58:35-41.

42. Seaman $P$, Edgar $F$, Ikegwuonu $T$. The role of alcohol price in young adult drinking cultures in Scotland. Drugs Educ Prev Policy 2013;20:278-85.

43. Wagenaar AC, Salois MJ, Komro KA. Effects of beverage alcoho price and tax levels on drinking: a meta-analysis of 1003 estimates from 112 studies. Addiction 2009;104:179-90.

44. Wagenaar AC, Tobler AL, Komro KA. Effects of alcohol tax and price policies on morbidity and mortality: a systematic review. Am J Public Health 2010;100:2270-8.

45. Angus C, Holmes J, Pryce R, et al. Model-based appraisal of the comparative impact of minimum unit pricing and taxation policies in Scotland an adaptation of the Sheffield Alcohol Policy Model version 3. ScHARR, University of Sheffield, 2016 April (cited 7 April 2016). https://www.shef.ac.uk/polopoly_fs/1.565373!/file/Scotland_report_ 2016.pdf

46. Booth A, Brennan A, Meier PS, et al. Independent review of the effects of alcohol pricing and promotion: part A-systematic reviews. Project report for the Department of Health September 2008. ScHARR University of Sheffield, 2008.

47. Brennan A, Purshouse R, Taylor $\mathrm{K}$, et al. Independent review of the effects of alcohol pricing and promotion: part B. Modelling the potential impact of pricing and promotion policies for alcohol in England: results from the Sheffield Alcohol Policy Model version 2008(1-1). ScHARR University of Sheffield, 2008. https://www.shef. ac.uk/polopoly_fs/1.95621!/file/PartB.pdf

48. Hill McManus D, Brennan A, Stockwell T, et al. Model-based appraisal of alcohol minimum pricing in Ontario and British Columbia: a Canadian adaptation of the Sheffield Alcohol Policy Model version 2. 2012. http://www.uvic.ca/research/centres/carbc/ assets/docs/report-model-based-appraisal.pdf

49. Institute for Fiscal Studies. The impact of Introducing a minimum price on alcohol in Britain. IFS Briefing Note BN109. 2010. https:// www.ifs.org.uk/publications/5287 (accessed 27 March 2017)

50. Institute for Fiscal Studies. Price-based measures to reduce alcohol consumption. IFS Briefing Note BN138. 2013. https://www.ifs.org.uk/ publications/6644 (accessed 27 March 2017)

51. Meng Y, Purshouse R, Brennan A, et al. Model-based appraisal of alcohol minimum pricing and off-licensed trade discount bans in Scotland using the Sheffield Alcohol Policy Model (v.2): second update based on newly available data. 2010. http://www.shef.ac.uk/ polopoly_fs/1.96510!/file/scotlandupdate.pdf

52. Rabinovich L, Brutscher PB, de Vries $\mathrm{H}$, et al. The affordability of alcoholic beverages in the European Union. 2012. http://ec.europa eu/health/ph_determinants/life_style/alcohol/documents/alcohol_ rand en.pdf (accessed 27 March 2017)

53. Gallet CA. The demand for alcohol: a meta-analysis of elasticities. Rochester, NY: Social Science Research Network, 2007 May (cited 6 January 2016). Report No.: ID 985689. http://papers.ssrn.com/ abstract $=985689$.

54. Gruenewald PJ, Ponicki WR, Holder HD, et al. Alcohol prices, beverage quality, and the demand for alcohol: quality substitutions and price elasticities. Alcohol Clin Exp Res 2006;30:96-105.

55. Ally AK, Meng $Y$, Chakraborty R, et al. Alcohol tax pass-through across the product and price range: do retailers treat cheap alcohol differently? Addiction 2014;109:1994-2002.

56. Rabinovich L, Hunt $P$, Staetsky $L$, et al. Further study on the affordability of alcoholic beverages in the EU [Internet]. 2012 (cited 17 February 2017). http://www.rand.org/pubs/technical_reports/ TR1203.html

57. Burton R, Henn C, Lavoie D, et al. A rapid evidence review of the effectiveness and cost-effectiveness of alcohol control policies: an English perspective. Lancet 2016. 